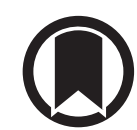

CrossMark

\section{Anti-IL5 and anti-IL5R $\alpha$ therapy for clinically significant bronchiectasis with eosinophilic endotype: a case series}

\author{
To the Editor:
}

Bronchiectasis is a chronic and often progressive disease, which frequently is associated with significant symptom burden, requiring intensive treatment. Regardless of the multiple potential underlying aetiologies, the vicious cycle of airway inflammation, structural airway damage, impaired mucus clearance and airway pathogen acquisition is the crucial pathogenic pathway for the progression of disease [1].

Although inflammation in bronchiectasis has been classically regarded as neutrophilic, it is increasingly recognised that eosinophils may play a role in the disease. Allergic bronchopulmonary aspergillosis (ABPA) is an example of a common cause of bronchiectasis that is primarily eosinophilic. Small studies suggest that a significant number of patients have airway eosinophilia even after exclusion of patients with underlying ABPA and asthma. TsikrikA et al. [2] found that among 40 patients with bronchiectasis excluding asthma and ABPA, 30\% in total had sputum eosinophilia, defined as $>3 \%$ cells. This supports the possibility that there may be an eosinophilic endotype of bronchiectasis, although published data on the prevalence and clinical significance of airway and systemic eosinophilia in bronchiectasis remain limited $[2,3]$.

Mepolizumab, a humanised monoclonal antibody, reduces eosinophil counts in blood and tissues by blocking interleukin (IL)-5, a key eosinophil cytokine, and preventing its binding to eosinophil surface receptors [4]. In patients with severe eosinophilic asthma, add-on mepolizumab treatment resulted in a decrease of exacerbation frequency, reduced symptom burden, improved of quality of life (QoL) and, moreover, had an oral corticosteroid (OCS)-sparing effect [4-8].

The use of mepolizumab for eosinophilic chronic obstructive pulmonary disease (COPD) was associated with a lower annual rate of moderate or severe exacerbations $[9,10]$. Benralizumab is a biological drug blocking the $\alpha$-chain of the IL-5 receptor (IL5R $\alpha$ ), which leads to an antibody-dependent cell-mediated cytotoxicity. The use of benralizumab resulted in a reduction in exacerbations and OCS use in patients with severe eosinophilic asthma [11], but add-on benralizumab was not associated with a lower annualised rate of COPD exacerbations [12]. Overall, there is a considerable overlap between different eosinophilic diseases (allergic and eosinophilic asthma, eosinophilic granulomatosis with polyangiitis (EGPA), allergic bronchopulmonary aspergillosis/mycosis (ABPA/M)) as well as conditions that may be associated with significant peripheral eosinophilia (asthma-COPD overlap syndrome (ACOS), eosinophilic COPD). It has been recognised that not only the clinical phenotype of severe asthma may have different underlying endotypes [13]; recently, one pilot study of four patients with severe uncontrolled asthma and concomitant bronchiectasis showed the efficacy of mepolizumab in terms of exacerbations, forced expiratory volume in $1 \mathrm{~s}\left(\mathrm{FEV}_{1}\right)$ in blood as well as sputum and nasal eosinophils [14]. The aim of the present retrospective case series was to study the effect of anti-eosinophilic therapy using anti-IL5 and anti-IL5 $\alpha$ monoclonal antibodies in patients with the primary diagnosis of bronchiectasis and a consistent eosinophil endotype defined by a reproducible peripheral eosinophila $\geqslant 300$ cells $\mu \mathrm{L}^{-1}$ at baseline or before initiation of long-term OCS therapy.

Overall, 450 patients, accounting for 1392 consultations, had been in regular follow-up at the Adult Bronchiectasis Clinic of the Hannover Medical School (Hannover, Germany) between January 2016 and

@ERSpublications

Patients with clinically significant bronchiectasis featuring an eosinophilic inflammatory endotype who were treated with add-on mepolizumab or benralizumab showed a significant improvement of $\mathrm{FEV}_{1}$, symptom burden and quality of life http://bit.ly/2AZvBLm

Cite this article as: Rademacher J, Konwert S, Fuge J, et al. Anti-IL5 and anti-IL5R $\alpha$ therapy for clinically significant bronchiectasis with eosinophilic endotype: a case series. Eur Respir J 2020; 55: 1901333 [https:// doi.org/10.1183/13993003.01333-2019]. 
October 2018. Of those, $11 \%$ had blood eosinophils $\geqslant 300$ cells $\mu \mathrm{L}^{-1}$ during stable state. In 21 patients of this subgroup we decided to initiate therapy with an anti-IL5 or anti-ILR $\alpha$ antibody due to refractory disease despite optimised maintenance therapy, including recommended vaccinations, pulmonary rehabilitation, daily chest physiotherapy with adjunct nebulised mucolytics and triple inhaled therapy consisting of high-dose inhaled corticosteroids and long-acting dual bronchodilator therapy. All patients were aged $>18$ years, had characteristic symptoms of clinically significant bronchiectasis, including cough and sputum production on most days of the week as well as a history of exacerbations, computed tomography-confirmed bronchiectasis and were referred to our adult bronchiectasis clinic by chest physicians due to the diagnosis of bronchiectasis. In addition, all subjects had frequent or severe pulmonary exacerbations requiring OCS in the previous year, chronic airflow limitation, reduced QoL and persistent as well as reproducible peripheral eosinophilia $\geqslant 300$ cells $\mu \mathrm{L}^{-1}$ at baseline or before initiation of long-term OCS therapy, respectively.

Overall, 12 bronchiectasis patients receiving mepolizumab and nine patients receiving benralizumab had complete follow-up data available after 3 and 6 months of therapy, including the number of exacerbations in the previous 12 months, lung function tests, eosinophil blood count, modified Medical Research Council (mMRC) dyspnoea scale, 24-h sputum volume and overall QoL measured using a visual analogue scale (VAS; score 0-10). Of those, $10(48 \%)$ were female; median (interquartile range (IQR)) age was 55 (51-58) years; median (IQR) time of chronic respiratory symptoms was 18.6 (5.8-38.1) years; and median (IQR) duration of known bronchiectasis was 2.8 (1.1-13.1) years. 14 (67\%) patients were on long-term OCS treatment, three patients received anti-inflammatory azithromycin maintenance therapy and four patients had obtained omalizumab for allergic asthma and/or ABPA before treatment. The most common pathogens in sputum culture were Aspergillus fumigatus $(\mathrm{n}=4)$, followed by Staphylococcus aureus $(\mathrm{n}=3)$, Haemophilus influenzae $(\mathrm{n}=3)$ and Pseudomonas aeruginosa $(\mathrm{n}=2)$. Eight subjects were former smokers; there were no active smokers in the study group. After comprehensive and thorough diagnostic work-up according to current guidelines $[15,16]$, including bronchodilator reversibility testing, exhaled nitric oxide fraction, measurement of nasal nitric oxide, sweat test and panel genetics for CFTR and primary ciliary dyskinesia (PCD) pathogenic genetic variants if indicated, the aetiology of bronchiectasis was severe and refractory eosinophilic asthma $(n=4)$, severe allergic and eosinophilic asthma $(n=4), A B P A / M(n=4)$, idiopathic $(n=2)$, ACOS $(n=1)$, EGPA $(n=1)$ and PCD $(n=1)$ as determined by the treating physician. In the remaining four subjects no distinction could be made between severe and refractory eosinophilic asthma and EGPA according to established EGPA criteria, as disease manifestations were limited to the upper and lower airways (with no antineutrophil cytoplasmic antibodies detectable, history of asthma, $\geqslant 10 \%$ eosinophils on differential leukocyte count, paranasal sinus abnormalities and radiographical detection of transient pulmonary opacities or (transbronchial or sinonasal) biopsy containing a blood vessel showing the accumulation of eosinophils in extravascular areas) [17]. We refrained from performing bronchial challenge testing as all patients showed mild to very severe chronic airflow limitation at baseline, with 19 (90\%) out of 21 subjects already showing at least moderate chronic airflow limitation.

Mepolizumab $100 \mathrm{mg}$ (every 4 weeks) and benralizumab $30 \mathrm{mg}$ (every 4 weeks for the first three doses, then every 8 weeks) were administered subcutaneously, as licensed for use in severe eosinophilic asthma. As all subjects had refractory disease despite optimised maintenance therapy, both drugs were used as part of a targeted and individualised treatment attempt, with no ethical consultation required. SPSS Statistics (version 25.0; IBM Corporation, Armonk, NY, USA) and STATA (version 13.0; StataCorp, College Station, TX, USA) statistical software programmes were used to analyse the data. Comparisons between different time points were performed using the non-parametric Wilcoxon signed-rank test. A two-tailed p-value of $<0.05$ was considered statistically significant.

After 6 months of treatment with anti-IL5 and anti-IL-5R $\alpha$ therapy the median (IQR) FEV $_{1}$ increased from $53(40-69) \%$ predicted to $68(38-94) \%$ pred (figure $1 \mathrm{a} ; \mathrm{p}=0.033)$. In the same period, the median (IQR) annualised exacerbation rate improved from 3 (1-6) exacerbations at baseline to 1 (0-3) (figure $1 \mathrm{~b}$; $\mathrm{p}=0.059)$. Similar results were observed for the median (IQR) score on the mMRC dyspnoea scale, which decreased from $2(1-3)$ to $0(0-1)$ (figure $1 \mathrm{c} ; \mathrm{p}=0.008)$. As a patient-reported outcome, the median (IQR) QoL (VAS) improved significantly from 4 (3-6) to 7 (5-8) (figure $1 \mathrm{~d} ; \mathrm{p}=0.002$ ), while the median (IQR) eosinophil count decreased significantly from $800(550-1240)$ cells $\mu \mathrm{L}^{-1}$ to $100(0-100)$ cells $\mu \mathrm{L}^{-1}$ (figure $1 \mathrm{e} ; \mathrm{p}<0.001)$ and the median 24 -h sputum volume (IQR) decreased from $10(8-25) \mathrm{mL}$ to $8(0-14) \mathrm{mL}$ (figure 1f; $\mathrm{p}=0.009$ ). In all 14 patients on long-term OCS therapy an OCS-sparing effect was observed with therapy. Nine out of 14 patients were able to cease OCS therapy, while five patients were able to reduce the OCS dose (prednisone equivalent median (IQR) 6.5 (4.3-7.8) $\mathrm{mg}$ at baseline versus 3.8 (1.8-5) $\mathrm{mg}$ after 6 months; $\mathrm{p}=0.042$ ). Using improvement of $\mathrm{FEV}_{1}$, decrease of blood eosinophils and improvement of any subjective measure (mMRC or QoL) as response criteria $13(62 \%)$ out of 21 patients were classified as responders to anti-eosinophilic therapy [18]. 

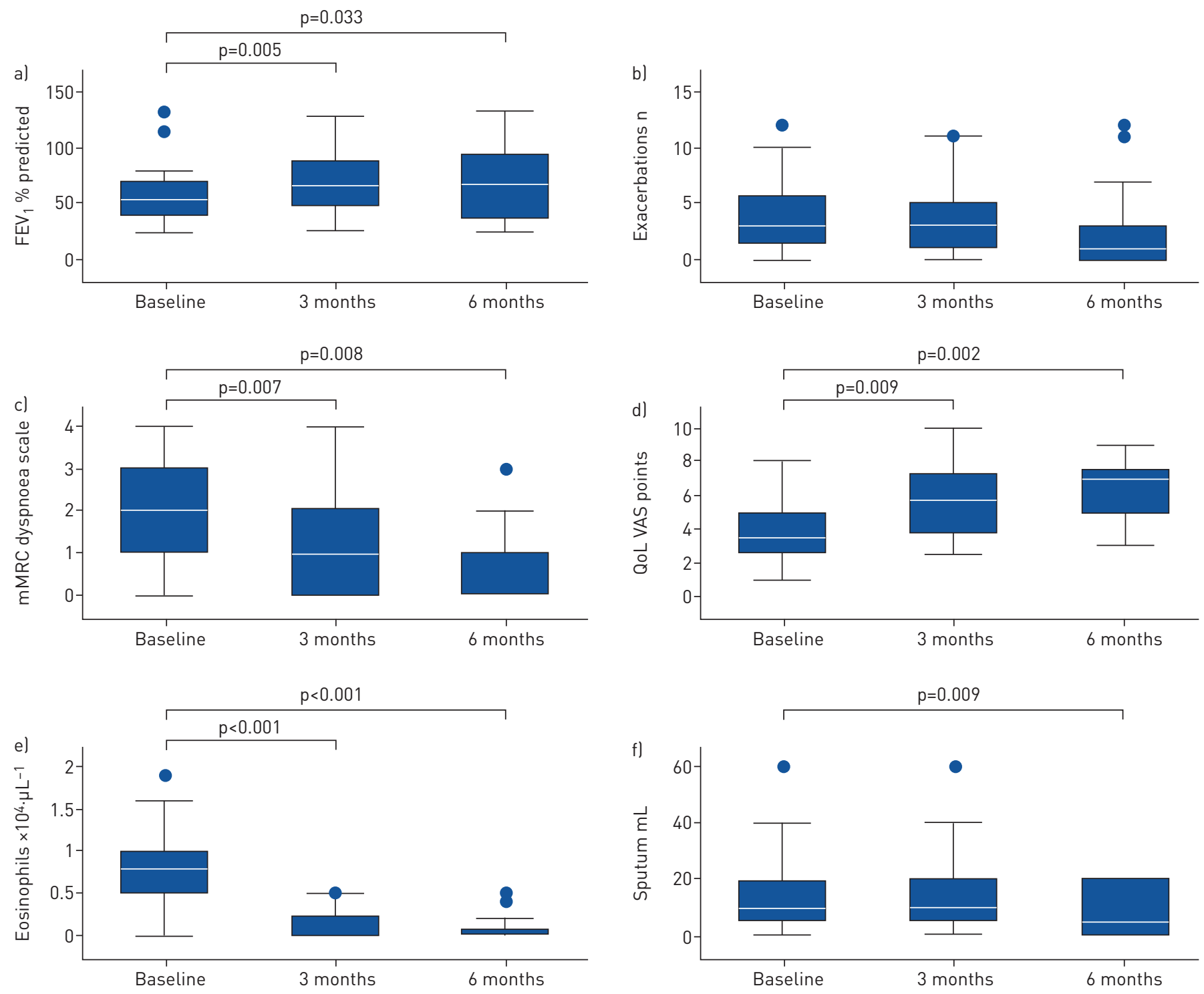

FIGURE 1 a) Forced expiratory volume in $1 \mathrm{~s}\left(\mathrm{FEV}_{1}\right)$; b) annualised exacerbation frequency; c) modified Medical Research Council (mMRC) dyspnoea scale; d) quality of life (QoL); e) eosinophils; and f) 24-h sputum volume at baseline and after 3 and 6 months of treatment. VAS: visual analogue scale.

Overall, mepolizumab and benralizumab were well tolerated. One patient with underlying OCS-dependent eosinophilic asthma experienced bilateral pneumonia requiring hospitalisation 10 days after first application of mepolizumab. Another patient with EGPA developed chronic parotitis after being treated with mepolizumab for several months. However, in both subjects these events were rated by the treating physician as unrelated to treatment with mepolizumab, but rather related to the underlying condition.

Overall, patients with clinically significant bronchiectasis featuring an eosinophilic inflammatory endotype, who did not respond to standard bronchiectasis treatments and were treated with add-on mepolizumab or benralizumab showed a significant reduction of blood eosinophils as well as a significant improvement of $\mathrm{FEV}_{1}$, symptom burden and QoL. Moreover, we observed a trend towards a reduced annualised exacerbation frequency. Our results suggest that monoclonal anti-IL5 or anti-IL5R $\alpha$ antibodies may be promising add-on anti-eosinophilic treatment options for this extensively pretreated patient population.

Our results are in line with earlier studies showing a reduction of exacerbations with the use of mepolizumab or benralizumab in patients with eosinophilic asthma and eosinophilic COPD [5, 8, 10, 11, 19]. In addition, Chupp et al. [6] described an increase in QoL and a decrease in symptom burden, similar to our findings. Our study has several limitations. First, the sample size was small and the observation period was comparatively short so that exacerbation frequency had to be annualised. However, it might be expected that our results would have been even more impressive after 12 months of treatment. Second, the 
measurement of QoL by VAS is not validated for patients with bronchiectasis. Nevertheless, a similar VAS has previously been used to quantify symptoms for lower respiratory tract infections in bronchiectasis and showed excellent validity, reliability and responsiveness in assessment of overall symptom burden [20]. All of the patients in this study had a primary diagnosis of bronchiectasis, but there was clearly evidence of overlap with other eosinophil diseases including asthma, ABPA and EGPA. This is not surprising, as overlap between bronchiectasis and other airway diseases is common and we nevertheless demonstrated effectiveness of anti-IL-5 therapy in a cohort including patients with idiopathic and PCD-related bronchiectasis without evident overlap. Finally, the findings of our case study are preliminary and it remains to be determined whether the effects are sustained over time.

Nevertheless, our findings emphasise the importance of recognising the underlying inflammatory endotype as a prerequisite for successful treatment in line with the concept of treatable traits [21]. Bronchiectasis has always been perceived as a purely neutrophilic disease. In this regard, our findings challenge traditional definitions of airway diseases. However, as inflammation persists, disease progresses and tissue damage as well as further complications such as chronic infections arise, the origin of bronchiectasis can be impossible to establish beyond any doubt. Given the fact that the majority of recent clinical trials in bronchiectasis (which mainly addressed neutrophilic inflammation and infection) have been unsuccessful, we propose that there are shared biological mechanisms between bronchiectasis and several eosinophilic diseases in many patients [22-24].

In conclusion, a randomised controlled trial evaluating the use of a monoclonal anti-eosinophilic antibody as an anti-inflammatory add-on therapy in subjects with clinically significant bronchiectasis and eosinophilic endotype is warranted.

Jessica Rademacher ${ }^{1,2}$, Stefanie Konwert $^{1}$, Jan Fuge $\odot^{2}$, Sabine Dettmer ${ }^{2,3}$, Tobias Welte ${ }^{1,2}$ and Felix C. Ringshausen $\oplus^{1,2}$

${ }^{1}$ Dept of Respiratory Medicine, Hannover Medical School, Hannover, Germany. ${ }^{2}$ Biomedical Research in Endstage and Obstructive Lung Disease Hannover (BREATH), German Center for Lung Research (DZL), Hannover, Germany. ${ }^{3}$ Dept of Diagnostic and Interventional Radiology, Hannover Medical School, Hannover, Germany.

Correspondence: Jessica Rademacher, Dept of Respiratory Medicine, Hannover Medical School, Carl-Neuberg-Strasse 1, 30625 Hannover, Germany. E-mail: rademacher.jessica@mh-hannover.de

Received: 14 Feb 2019 | Accepted after revision: 05 Oct 2019

Conflict of interest: J. Rademacher reports grants and personal fees from Bayer Healthcare, Insmed and Grifols, personal fees from MSD Sharp and Dohme, AstraZeneca and Chiesi, outside the submitted work. S. Konwert has nothing to disclose. J. Fuge has nothing to disclose. S. Dettmer has nothing to disclose. T. Welte reports personal fees for advisory board work and lectures from AstraZeneca, GSK, Grifols, Insmed, Bayer and Novartis, during the conduct of the study; personal fees for advisory board work and lectures from Boehringer and Pfizer, outside the submitted work. F.C Ringshausen reports grants, personal fees and other from Bayer Healthcare, Insmed Germany and Novartis, grants and personal fees from Grifols, personal fees from MSD Sharp and Dohme, AstraZeneca Boehringer Ingelheim and Vortex, personal fees and other from Chiesi and Gilead, grants from Polyphor, grants and other from InfectoPharm, other from Abbott, Pfizer, Oxycare, Heinen and Löwenstein, MSD, Vertex, Parion, Cetaxsys, Corbus, GSK, PARI, APOSAN and Zambon, outside the submitted work.

\section{References}

1 Chalmers JD, Aliberti S, Blasi F. Management of bronchiectasis in adults. Eur Respir J 2015; 45: 1446-1462.

2 Tsikrika S, Dimakou K, Papaioannou AI, et al. The role of non-invasive modalities for assessing inflammation in patients with non-cystic fibrosis bronchiectasis. Cytokine 2017; 99: 281-286.

3 Abo-Leyah H, Finch S, Keir H, et al. Peripheral blood eosinophilia and clinical phenotype in bronchiectasis. Eur Respir J 2018; 52: Suppl. 62, PA2665.

4 Varricchi G, Bagnasco D, Borriello F, et al. Interleukin-5 pathway inhibition in the treatment of eosinophilic respiratory disorders: evidence and unmet needs. Curr Opin Allergy Clin Immunol 2016; 16: 186-200.

5 Ortega HG, Yancey SW, Mayer B, et al. Severe eosinophilic asthma treated with mepolizumab stratified by baseline eosinophil thresholds: a secondary analysis of the DREAM and MENSA studies. Lancet Respir Med 2016; 4: 549-556.

6 Chupp GL, Bradford ES, Albers FC, et al. Efficacy of mepolizumab add-on therapy on health-related quality of life and markers of asthma control in severe eosinophilic asthma (MUSCA): a randomised, double-blind, placebo-controlled, parallel-group, multicentre, phase 3b trial. Lancet Respir Med 2017; 5: 390-400.

7 Bel EH, Wenzel SE, Thompson PJ, et al. Oral glucocorticoid-sparing effect of mepolizumab in eosinophilic asthma. N Engl J Med 2014; 371: 1189-1197.

8 Haldar P, Brightling CE, Hargadon B, et al. Mepolizumab and exacerbations of refractory eosinophilic asthma. N Engl J Med 2009; 360: 973-984.

9 Sciurba FC, Bradford ES, Pavord ID. Mepolizumab for eosinophilic COPD. N Engl J Med 2018; 378: $681-683$.

10 Pavord ID, Chanez P, Criner GJ, et al. Mepolizumab for eosinophilic chronic obstructive pulmonary disease. N Engl J Med 2017; 377: 1613-1629.

11 FitzGerald JM, Bleecker ER, Nair P, et al. Benralizumab, an anti-interleukin-5 receptor $\alpha$ monoclonal antibody, as add-on treatment for patients with severe, uncontrolled, eosinophilic asthma (CALIMA): a randomised, double-blind, placebo-controlled phase 3 trial. Lancet 2016; 388: 2128-2141. 
12 Criner GJ, Celli BR, Brightling CE, et al. Benralizumab for the prevention of COPD exacerbations. $N$ Engl J Med 2019; 381: 1023-1034.

13 Schleich FN, Manise M, Sele J, et al. Distribution of sputum cellular phenotype in a large asthma cohort: predicting factors for eosinophilic vs neutrophilic inflammation. BMC Pulm Med 2013; 13: 11.

14 Carpagnano GE, Scioscia G, Lacedonia D, et al. Severe uncontrolled asthma with bronchiectasis: a pilot study of an emerging phenotype that responds to mepolizumab. J Asthma Allergy 2019; 12: 83-90.

15 Polverino E, Goeminne PC, McDonnell MJ, et al. European Respiratory Society guidelines for the management of adult bronchiectasis. Eur Respir J 2017; 50: 1700629.

16 Hill AT, Sullivan AL, Chalmers JD, et al. British Thoracic Society guideline for bronchiectasis in adults. BMJ Open Respir Res 2018; 5: e000348.

17 Masi AT, Hunder GG, Lie JT, et al. The American College of Rheumatology 1990 criteria for the classification of Churg-Strauss syndrome (allergic granulomatosis and angiitis). Arthritis Rheum 1990; 33: 1094-1100.

18 Drick N, Seeliger B, Welte T, et al. Anti-IL-5 therapy in patients with severe eosinophilic asthma - clinical efficacy and possible criteria for treatment response. BMC Pulm Med 2018; 18: 119.

19 Bleecker ER, FitzGerald JM, Chanez P, et al. Efficacy and safety of benralizumab for patients with severe asthma uncontrolled with high-dosage inhaled corticosteroids and long-acting $\beta_{2}$-agonists (SIROCCO): a randomised, multicentre, placebo-controlled phase 3 trial. Lancet 2016; 388: 2115-2127.

20 Altenburg J, Wortel K, de Graaff CS, et al. Validation of a visual analogue score (LRTI-VAS) in non-CF bronchiectasis. Clin Respir J 2016; 10: 168-175.

21 Boaventura R, Sibila O, Agusti A, et al. Treatable traits in bronchiectasis. Eur Respir J 2018; 52: 1801269.

22 De Soyza A, Aksamit T, Bandel T-J, et al. RESPIRE 1: a phase III placebo-controlled randomised trial of ciprofloxacin dry powder for inhalation in non-cystic fibrosis bronchiectasis. Eur Respir J 2018; 51: 1702052.

23 Aksamit T, De Soyza A, Bandel T-J, et al. RESPIRE 2: a phase III placebo-controlled randomised trial of ciprofloxacin dry powder for inhalation in non-cystic fibrosis bronchiectasis. Eur Respir J 2018; 51: 1702053.

24 Chalmers JD, Chotirmall SH. Bronchiectasis: new therapies and new perspectives. Lancet Respir Med 2018; 6: $715-726$ 